\title{
Association of delivery procedure with APGAR scores among neonates born to healthy Pakistani mothers: a pilot study
}

\section{[version 1; peer review: 1 not approved]}

\author{
Muhammad Ali Khalid (D1), Rida Ghani1, Muhammad Fahad Khalid2, \\ Muhammad Saad Malik', Ahmed Waqas (iD) \\ ${ }^{1} \mathrm{CMH}$ Lahore Medical College \& Institute of Dentistry, Lahore, Cantt, Pakistan \\ ${ }^{2}$ Rawalpindi Medical University, Rawalpindi, Pakistan
}

V1 First published: 21 Mar 2018, 7:346

https://doi.org/10.12688/f1000research.13784.1

Latest published: 21 Mar 2018, 7:346

https://doi.org/10.12688/f1000research.13784.1

\section{Abstract}

Background: The present study explores the factors associated with poor APGAR scores among singletons born to healthy Pakistani mothers.

Methods: This cross-sectional study was conducted at a Tara Urea Medical Center, Iskandarabad Colony, district Mianwali, Pakistan from April 1 to August 30, 2017. Data was collected using a preformed proforma by a gynecologist and pediatrician during the birth procedure. The questionnaire comprised of two sections including neonatal and maternal characteristics. All data were analyzed in SPSS v.20.

Results: Regression analysis revealed that vaginal deliveries were associated with higher APGAR scores at five minutes than those delivered by cesarean section. However, maternal age and BMI and weight of the baby did not yield significant association with APGAR scores at five minutes. APGAR scores assessed at one minute were significantly associated with weight of the neonate.

Conclusion: APGAR scores of the neonates at birth are significantly associated with birth procedures. Therefore, birth procedure should be selected and managed effectively to reduce the risk of low APGAR scores.

Keywords

APGAR, neonate, factors, predictors

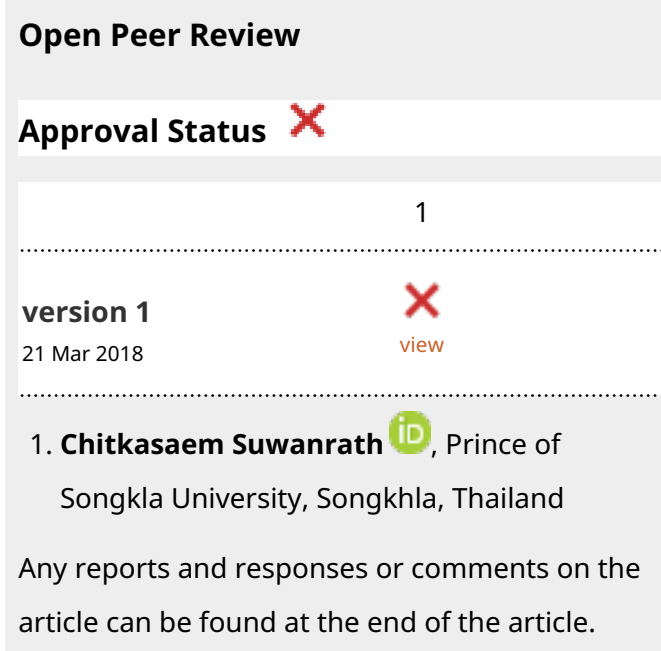

article can be found at the end of the article. 
Corresponding author: Muhammad Ali Khalid (alikhalid1995@gmail.com)

Author roles: Khalid MA: Conceptualization, Data Curation, Formal Analysis, Supervision, Writing - Original Draft Preparation; Ghani R: Investigation, Methodology, Project Administration, Writing - Original Draft Preparation, Writing - Review \& Editing; Khalid MF: Investigation, Writing - Original Draft Preparation, Writing - Review \& Editing; Malik MS: Investigation, Writing - Original Draft Preparation, Writing - Review \& Editing; Waqas A: Writing - Review \& Editing

Competing interests: No competing interests were disclosed.

Grant information: The author(s) declared that no grants were involved in supporting this work.

Copyright: @ 2018 Khalid MA et al. This is an open access article distributed under the terms of the Creative Commons Attribution License, which permits unrestricted use, distribution, and reproduction in any medium, provided the original work is properly cited. Data associated with the article are available under the terms of the Creative Commons Zero "No rights reserved" data waiver (CC0 1.0 Public domain dedication).

How to cite this article: Khalid MA, Ghani R, Khalid MF et al. Association of delivery procedure with APGAR scores among neonates born to healthy Pakistani mothers: a pilot study [version 1; peer review: 1 not approved] F1000Research 2018, 7:346

https://doi.org/10.12688/f1000research.13784.1

First published: 21 Mar 2018, 7:346 https://doi.org/10.12688/f1000research.13784.1 


\section{Introduction}

In recent years, much has been published regarding the morbidity and mortality among newborns. However, most research studies are limited to the paradigm of Western countries. A limited contribution in terms of large scale prevalence studies, and culture and resource sensitive interventions have been made from the developing and third world countries. This is a major public health concern because the majority of newborn deaths occur in poorly resourced developing world. According to the World Health Organization (WHO), 2.6 million neonates died in 2016, accounting for a staggering 7,000 newborn deaths daily, with 1 million dying on the first day of life. Therefore, it is of paramount importance to assess the health status of the neonate during the birth procedure using validated methods such as the APGAR scoring system.

The APGAR score (AS) is validated, reliable and one of the most widely used clinical tools for evaluation of health status of the newborn immediately after birth ${ }^{1}$. The APGAR scoring system was originally developed by Virginia Apgar (1953) based on five characteristics including heart rate of the newborn, respiratory effort, muscle tone, irritability, and color ${ }^{1}$. Thus, APGAR scores provide a convenient method to recognize life threatening situations for the newborn and their subsequent response to resuscitation techniques ${ }^{2}$. A low APGAR score is also a significant predictor of neonatal mortality, long term neurological development, IQ scores, motor impairment, cerebral palsy, mental retardation, epilepsy and poor academic performance $^{3,4}$.

Previous literature has shown that APGAR scores of neonates is dependent on a number of factors including maternal age, body mass index, education level, parity, previous miscarriages, emotional well-being, use of nicotine and antidepressants, gestational age, medical comorbidities, and multiple pregnancies $^{5}$. Method of delivery of the newborn has also been demonstrated as a significant predictor of APGAR scores among the newborns.

However, the evidence regarding the association between mode of delivery and APGAR scores is controversial. For instance, Kilsztajn and colleagues reported that compared to vaginal deliveries, neonates born through c-section had higher APGAR scores $^{6}$. However, they found that this association was spurious and driven by confounding with indication. A few studies also stratify the risk of low APGAR scores according to the urgency of birth procedure. For instance, Lai et al., reported that only emergency cesarean and instrumental vaginal deliveries are associated with low to moderate APGAR scores ${ }^{2}$. Similar results have been corroborated by Johnson et al. and Prior et al. in their studies exploring predictive factors of neonatal outcomes ${ }^{7,8}$. This variation in APGAR scores among neonates born through different birth procedures is likely due to oxidative stress experienced by them, however, studies exploring this hypothesis have also yielded mixed results ${ }^{9-11}$.

In addition to delivery procedures, a number of other factors have been recognized as independent predictors of APGAR scores among neonates. These include non-cephalic presentation, intramuscular narcotic use, presence of meconium, nulliparity, preterm birth, body mass index, emotional distress, type of pregnancy, and antenatal healthcare ${ }^{2,5}$.

Due to paucity of data, the present study explores the factors associated with poor APGAR scores among singletons born to healthy Pakistani mothers.

\section{Methods}

This cross-sectional study was conducted at Tara Urea Medical Center, Iskandarabad Colony, district Mianwali and Majid Medical Complex, Lahore, both in Pakistan from April 1 to August 30, 2017. Participants undergoing birth procedures at this center were recruited randomly using an online computer software. Only those women were found eligible who had a term birth, no previous history of comorbid medical disorders and antepartum complications were included. All participants provided written informed consent and they were ensured anonymity and that no individual data would be disclosed. Ethical review was sought and granted by the research committee at the Tara Urea Medical Center, Iskandarabad Colony, district Mianwali, Pakistan (Reference \# IRB-001).

Data was collected using a preformed proforma by a gynecologist and pediatrician during the birth procedure. The questionnaire (Supplementary File 1) comprised of two sections including neonatal and maternal characteristics. Maternal characteristics included body mass index, age and the birth procedure used for delivering the newborn. Neonatal characteristics included gender, weight, admission to neonatal intensive care unit, complications during delivery, and APGAR scores at 1 and 5 minutes.

Using Gpower v3.1.7, a minimum sample size of 92 was considered to be appropriate for this study to achieve a power of $80 \%$ for an anticipated moderate effect size and five predictors. All data were analyzed using SPSS v.20 (IMB, Chicago, IL). Quantitative variables were presented as mean (SD) and categorical as frequency (\%). Normality of quantitative variables was assessed by visualizing histograms. Thereafter, linear regression analysis was run to explore the predictors of APGAR scores at one and five minutes. Both maternal and neonatal characteristics were introduced as independent variables in these models.

\section{Results}

Out of 100 women undergoing birth procedures, a total of 98 $(98 \%)$ agreed to participate in the study. There were 49 male (50\%) and 49 female (50\%) neonates. A majority of the neonates were born though supra-vaginal delivery $(67,68.4 \%)$ followed by cesarean section $(22,22.4 \%)$ and episiotomy $(9,9.2 \%)$. A total of six $(6.1 \%)$ neonates experienced complications during delivery and four $(4.1 \%)$ were admitted to neonatal intensive care unit. Mean weight of mothers was 72.51 (6.32) kg, height 160.85 (4.64) cm, age 23.94 (2.81) years and BMI 28.06 (2.25). Mean weight of the neonates was $2.99(0.25) \mathrm{kg}$, APGAR score at one minute 6.18 (1.37) and APGAR score at five minutes was 9.67 (0.69). 
Linear regression analysis delineating predictors of APGAR scores at one minute explained only $5.3 \%$ variation. According to it, maternal age, BMI and type of delivery procedures were not associated with APGAR scores assessed at one minute after delivery. However, it was inversely associated with weight of the neonate.

Regression modelling for APGAR scores assessed at five minutes explained $70.60 \%$ of variation in it. According to it, vaginal deliveries and higher maternal BMI were associated with higher APGAR scores at five minutes than those delivered by cesarean section. However, maternal age and weight of the baby did not yield significant associations with APGAR scores at five minutes. Detailed results are presented in Table 1.

\section{Dataset 1. APGAR factors}

http://dx.doi.org/10.5256/f1000research.13784.d198118

Coding Scheme: Gender: $1=$ Male, $2=$ female; Mode of delivery: 1= C-section, 2= supra-vaginal delivery, 3= SVD with episiotomy; Admission to $\mathrm{NICU}=1=\mathrm{yes}, 2=\mathrm{no}$; Complications during delivery= $1=\mathrm{yes}, 2=$ no. Mode of delivery binary $=1=\mathrm{C}$-section, $2=$ other

Table 1. Predictors of APGAR scores assessed at one and five minutes $(n=98)$.

\begin{tabular}{|l|r|r|r|r|r|r|}
\hline \multicolumn{7}{|c|}{ APGAR scores at five minutes } \\
\hline Variables & B & Std. Error & Beta & t-value & P-value \\
\hline Constant & 0.847 & 0.098 & & 8.615 & $<0.001$ \\
\hline Maternal age & -0.069 & 0.048 & -0.095 & -1.437 & 0.154 \\
\hline $\begin{array}{l}\text { Weight of the } \\
\text { neonate }\end{array}$ & -0.068 & 0.055 & -0.072 & -1.240 & 0.218 \\
\hline \begin{tabular}{l|r|r|r|r|r|} 
Birth procedure \\
Maternal BMI
\end{tabular} & 0.064 & 0.005 & 0.794 & 11.941 & $<0.001$ \\
\hline & APGAR scores at one minute & & \\
\hline Constant & 1.121 & 0.564 & & 1.989 & 0.050 \\
\hline Maternal age & -0.016 & 0.276 & -0.007 & -0.057 & 0.955 \\
\hline $\begin{array}{l}\text { Weight of the } \\
\text { neonate }\end{array}$ & -0.686 & 0.315 & -0.227 & -2.176 & 0.032 \\
\hline $\begin{array}{l}\text { Birth procedure } \\
\text { Maternal BMI }\end{array}$ & -0.047 & 0.062 & 0.031 & -0.185 & -1.554 & 0.124 \\
\hline
\end{tabular}

$\mathrm{BMI}=$ Body mass index

Std. Error $=$ Standard Error

\section{Discussion}

In present study, delivery by cesarean section and low maternal BMI was associated with low APGAR scores assessed at five minutes. Whereas, the APGAR scores at one minute was significantly associated with weight of the neonate.

Our research evidence has several limitations and therefore, should be generalized with caution. The cross-sectional nature of the study design limits inferences regarding causality and temporality. Moreover, a small sample size $(n=98)$ limits the statistical power of this study, therefore, future studies should include greater study samples.

In conclusion, a newborn's health status is associated with the birth procedure as well as the birth-weight. These results have several implications for neonatal health in Pakistan on designing culture sensitive policies and educational interventions in antenatal care and preference for birthing procedures among expectant mothers as well as the practicing obstetricians and gynecologists. Expectant mothers should be delivered educational interventions during regular antenatal check-ups, aiming at improving overall health including an optimum weight of the fetus. Moreover, the rising preference of birth by cesarean sections among Pakistani expectant mothers should be discouraged.

\section{Data availability}

Dataset 1: APGAR factors 10.5256/f1000research.13784. d198118 12

Coding Scheme: Gender: $1=$ Male, $2=$ female; Mode of delivery: $1=\mathrm{C}$-section, 2 = supra-vaginal delivery, $3=$ SVD with episiotomy; Admission to NICU=1=yes, 2=no; Complications during delivery $=1=y e s, 2=$ no. Mode of delivery binary $=1=\mathrm{C}$-section, $2=$ other

\section{Competing interests}

No competing interests were disclosed.

\section{Grant information}

The author(s) declared that no grants were involved in supporting this work.

\section{Supplementary material}

Supplementary File 1 - Copy of study questionnaire

Click here to access the data. 
1. Casey BM, McIntire DD, Leveno KJ: The Continuing Value of the Apgar Score for the Assessment of Newborn Infants. N Engl J Med. 2001; 344(7): 467-71. PubMed Abstract | Publisher Full Text

2. Lai S, Flatley C, Kumar S: Perinatal risk factors for low and moderate five-minute Apgar scores at term. Eur J Obstet Gynecol Reprod Biol. 2017; 210: 251-6. PubMed Abstract | Publisher Full Text

3. Moore EA, Harris F, Laurens KR, et al:: Birth outcomes and academic achievement in childhood: A population record linkage study. J Early Child Res. 2014; 12(3): 234-50.

Publisher Full Text

4. Razaz N, Boyce WT, Brownell M, et al.: Five-minute Apgar score as a marker fo developmental vulnerability at 5 years of age. Arch Dis Child Fetal Neonatal Ed. 2016; 101(2): F114-20

PubMed Abstract | Publisher Full Text | Free Full Text

5. Almeida NKO, Pedreira CE, Almeida RMVR: Impact of maternal education level on risk of low Apgar score. Public Health. 2016; 140: 244-9. PubMed Abstract | Publisher Full Text

6. Kilsztajn S, de Souza Lopes E, Nunes do Carmo MS, et al.: [Apgar score associated with mode of delivery in São Paulo State, Brazil]. Cad Saude Publica. 2007; 23(8): 1886-92.

PubMed Abstract | Publisher Full Text
7. Johnson JH, Figueroa R, Carry D, et al.: Immediate maternal and neonatal effects of forceps and vacuum-assisted deliveries. Obstet Gynecol. 2004 103(3): 513-8.

PubMed Abstract | Publisher Full Text

8. Dunn L, Prior T, Greer R, et al:: Gender specific intrapartum and neonatal outcomes for term babies. Eur J Obstet Gynecol Reprod Biol. 2015; 185: 19-22. PubMed Abstract | Publisher Full Text

9. Raijmakers MT, Roes EM, Steegers EA, et al.: Umbilical glutathione levels are higher after vaginal birth than after cesarean section. J Perinat Med. undefined. degruyter.com [Internet]. 2003; 13(6), [cited 2018 Jan 11]. Publisher Full Text

10. Mehmetoglu I, Kart A, Caglayan O, et al:: Oxidative stress in mothers and their newborns in different types of labour. Turk J Med Sci. 2002; 32: 427-9. Reference Source

11. Fogel I, Pinchuk I, Kupfermine MJ, et al:: Oxidative stress in the fetal circulation does not depend on mode of delivery. Am J Obstet Gynecol. 2005; 193(1): 241-6. PubMed Abstract | Publisher Full Text

12. Khalid MA, Ghani R, Khalid MF, et al:: Dataset 1 in: Association of delivery procedure with APGAR scores among neonates born to healthy Pakistani mothers: a pilot study. F1000Research. 2018.

Data Source 


\section{Open Peer Review}

\section{Current Peer Review Status:}

\section{Version 1}

Reviewer Report 29 June 2022

https://doi.org/10.5256/f1000research.14983.r142234

(C) 2022 Suwanrath C. This is an open access peer review report distributed under the terms of the Creative Commons Attribution License, which permits unrestricted use, distribution, and reproduction in any medium, provided the original work is properly cited.

\section{Chitkasaem Suwanrath}

Prince of Songkla University, Songkhla, Thailand

This study has too small a sample size to determine predictive factors of Apgar scores. I have raised concerns on many points.

Method:

In the sample size calculation, the authors stated five predictors, please give details of the predictors

Which references the author used to calculate sample size $(N=92)$ ?

Are there any interventions in this study?

Were cesarean sections on demand allowed in the study hospitals?

Route of delivery is a categorical variable, not quantitative data. Is it appropriate to use linear regression?

Results:

What does supra-vaginal delivery mean? I have never heard this term?

Routes of delivery are classified as vaginal delivery and cesarean section. Episiotomy is in the category of vaginal delivery.

Since the sample size is small. Data distribution is unlikely normal. Data presented as median (min, max) or IQR may be more appropriate.

Please give details of the indication for cesarean section

Is the work clearly and accurately presented and does it cite the current literature? Partly 
Is the study design appropriate and is the work technically sound?

No

Are sufficient details of methods and analysis provided to allow replication by others? No

If applicable, is the statistical analysis and its interpretation appropriate?

No

Are all the source data underlying the results available to ensure full reproducibility? Yes

Are the conclusions drawn adequately supported by the results?

No

Competing Interests: No competing interests were disclosed.

Reviewer Expertise: Maternal fetal medicine

I confirm that I have read this submission and believe that I have an appropriate level of expertise to state that I do not consider it to be of an acceptable scientific standard, for reasons outlined above.

The benefits of publishing with F1000Research:

- Your article is published within days, with no editorial bias

- You can publish traditional articles, null/negative results, case reports, data notes and more

- The peer review process is transparent and collaborative

- Your article is indexed in PubMed after passing peer review

- Dedicated customer support at every stage

For pre-submission enquiries, contact research@f1000.com 